\title{
CNC-Milled Superhydrophobic Macroporous Monoliths for 3D Cell Culture
}

\author{
Gen Hayase ${ }^{\dagger} *$ and Daisuke Yoshino ${ }^{\ddagger}, *$ \\ tInternational Center for Materials Nanoarchitectonics, National Institute for Materials Science, 1-1 Namiki, Tsukuba, \\ Ibaraki, 305-0044, Japan. \\ ‡ Institute of Engineering, Tokyo University of Agriculture and Technology, 2-24-16 Naka-cho, Koganei, Tokyo, 184- \\ 8588, Japan. \\ macroporous monoliths, composites, CNC milling, superhydrophobic, multicellular tumor spheroids, 3D cell culture
}

\begin{abstract}
High-strength macroporous monoliths can be obtained by simply mixing boehmite nanofiber aqueous acetate dispersions with methyltrimethoxysilane. On the boehmite nanofiber-polymethylsilsesquioxane monoliths, we can fabricate structures smaller than a millimeter in size by computer numerical control (CNC) milling, resulting in a machined surface that is superhydrophobic and biocompatible. Using this strategy, we fabricated a superhydrophobic multiwell plate which holds water droplets to produce 3D cell culture environments for various cell types. We expect these superhydrophobic monoliths to have future applications in 3D tissue construction.
\end{abstract}

Macroporous monoliths with abundant pores, large specific surface area, and unique surface morphology have been studied as insulators, adsorbents, and catalyst carriers. ${ }^{1}$ In bioengineering, these materials have been put to practical use as sorbents in cell culture media and protein separation. ${ }^{2,3}$ Current applications use simple geometries, such as flat plates, disks, or rods. Additionally, macroporous materials with high porosity are generally brittle under tensile bending and twisting, and it is challenging to obtain microforms by machining after fabrication. When preparing sub-millimeter structures, it is common to transfer from a flexible template such as silicone. However, it is difficult to use this method in the case of high porosity macroporous materials because they are easily chipped during mold release. In recent years, the technological development of computer numerical control (CNC) milling desktop machines has made it possible to efficiently machine finely detailed features onto dense materials. ${ }^{4,5}$ Herein, we have directly processed high-strength macroporous materials by CNC milling to create advanced 3D geometries for cell culture.

Recently, we have developed macroporous monoliths with fiber-like structures that are less than $100 \mathrm{~nm}$ in diameter by coating and bonding boehmite nanofibers (BNFs, composed of $\mathrm{AlOOH}$ with a diameter of $4 \mathrm{~nm})^{6}$ with polyorganosilsesquioxane.7, 8 These "fiber-reinforced" materials, which can be prepared by the simple mixing of two liquids, have higher elasticity and bending strength than macroporous materials with similar skeletal diameters, and a superhydrophobic cut surface. Here, we have used this material to form intricate surface structures by CNC milling to test droplet handling and trapping. The composition of the macroporous material has been optimized from previous reports, and three samples were prepared with high shear density to improve mechanical strength. The following procedure was used to prepare the materials: (1) Mix $100 \mathrm{~mL}$ of a diluted BNF dispersed sol $\left(7.2 \cdot x^{-1} \mathrm{wt} \%\right.$ in acetic acid aqueous solution) and $100 \mathrm{~mL}$ of methyltrimethoxysilane (MTMS, $\mathrm{CH}_{3} \mathrm{Si}\left(\mathrm{OCH}_{3}\right)_{3}$ ) for $15 \mathrm{~min}$ and heating at $80{ }^{\circ} \mathrm{C}$ for one day. In this step, MTMS undergoes hydrolysis and polycondensation to form polymethylsilsesquioxane (PMSQ, $\mathrm{CH}_{3} \mathrm{SiO}_{1.5}$ ), which coats and binds BNFs into a gel; (2) wash with water and 2-propanol several times to exchange the internal liquid of the gel structure; and (3) dry at $60^{\circ} \mathrm{C}$ for $12 \mathrm{~h}$. This process yielded white xerogel panels, which we have named $\mathrm{X} x$ after the dilution ratio of the starting BNF sol, $x(=2.5,5,10)$. After the drying process, we repeatedly observed warping and cracking with the X2.5 and X5 materials, while the X10 sample was able to maintain large panels with high yield even under harsh drying conditions (Figure 1a). All three materials had a bulk density of about $0.29 \mathrm{~g} \mathrm{~cm}^{-3}$ (Table S1, Supporting Information), and the rod-like skeletal structure coarsened with increasing BNF concentration in the starting composition (Figures 1b,c, S1, S2, and Movie S1, Supporting Information). Uniaxial compression tests revealed that the BNF-PMSQ macroporous monoliths had the elasticity to recover their original shape from $25 \%$ compression, and the Young's modulus became lower with lower BNF concentration (Figure S3a, Supporting Information). From 3-point bending measurements, the BNF-PMSQ macroporous monoliths could withstand higher strains than the PMSQ porous materials with similar diameters (Figure S3b, Supporting Information $)^{9}$, which highlights one of the benefits of compounding with nanofibers. ${ }^{10}$ 

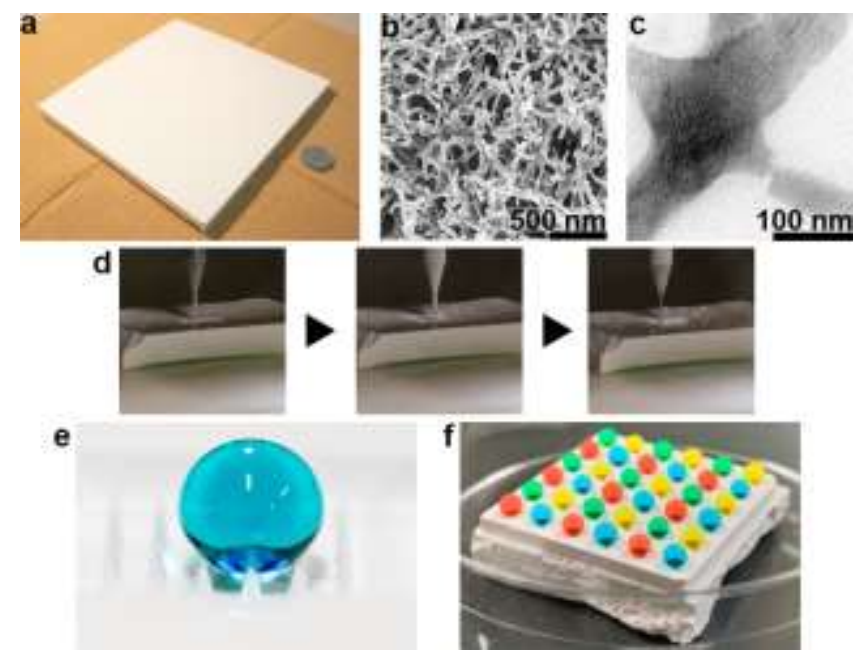

Figure 1. (a) Photograph (b) SEM and (c) TEM image of the X10 material. (d) Photographs of the X10 sample being CNC milled (see also Movie S2, Supporting Information). Colored water droplets (e) on microneedle arrays and (f) on a spheroid preparation plate formed by CNC milling. The design dimensions are shown in Figures S6 and S7 (Supporting Information).

Machining and application verification were carried out on the X10 material due to its high yield and favorable mechanical properties. First, the "flat" surface of the X10 sample was formed by CNC milling (Figure S4, Supporting Information). Water droplets formed a contact angle of $151^{\circ}$ with the surface, indicating a high degree of water repellency (Figure S5, Supporting Information). Next, we milled a microneedle consisting of a cone $500 \mu \mathrm{m}$ in diameter at the base and $1 \mathrm{~mm}$ high with a yield of over $95 \%$ (Figures $1 \mathrm{~d}$, S6, and Movie S2, Supporting Information). When water was dropped on this needle array, spherical droplets were retained (Figure 1e). By mixing aqueous solutions of different specific gravities on this substrate, we were able to form Janus-water droplets (Movie S3, Supporting Information). We also succeeded in creating grooves to arrange the droplets (Figure 1f). Since PMSQ has the same biocompatibility as other organic polysiloxanes, such as polydimethylsiloxane (PDMS), ${ }^{11}$ we attempted to use this substrate for 3D cell culture. In cancer research, 3D cell culture techniques have received attention as a pivotal technology to reproduce tumor microenvironments with 3D cell-cell and cell-matrix interactions. ${ }^{12}$ Although 2D surface, which allows for homogeneous cell culture, can induce cell-cell contact crosstalk, tumor cells are not in their normal state. Therefore, the 2Dcultured cells exhibit different responses from the 3D culture that is close to the actual in vivo environment. ${ }^{13-16} \mathrm{Tu}-$ mor spheroids are a commonly used 3D culture of cancer cells and can be formed by a variety of protocols, including ultra-low attachment plates, ${ }^{17}$ hanging drops, ${ }^{18}$ and magnetic levitation. ${ }^{19}$ However, these protocols require a certain amount of skill to produce spheroids consistently within the target size range.

Using our CNC milled microporous monoliths, we have created a simplified protocol that can form tumor spheroids with consistent size and shape. Although various methods of spheroid formation using hydrophobic substrates or microchannels have been reported, ${ }^{20-22}$ our process has advantages, such as the ability to obtain a near-spherical shape even when the diameter is large, depending on the computer-aided design. ${ }^{23}$ Spheroid formation was performed using the five steps outlined in Figure 2a: (1) Cell suspension was prepared by mixing ice-cold collagen solution $\left(4.0 \mathrm{mg} \mathrm{mL}^{-1}\right)$ and the harvested cells to give a final concentration. (2) The cell suspension was then dispensed onto the processed superhydrophobic multiwell plate. The dispensed volume corresponds to the diameter of the spheroid to be made. (3) The dispensed spherical cell suspension was incubated at $37{ }^{\circ} \mathrm{C}, 5 \% \mathrm{CO}_{2}$ for 30 to $60 \mathrm{~min}$. (4) After gelation, the spheroids were picked up with a micropipette and transferred to a cell culture plate or dish. (5) The spheroid became denser over a 3 to 5 -day incubation. The phase-contrast and fluorescence images of the spheroid were captured every day for 5 days using a wide-field fluorescence microscope.

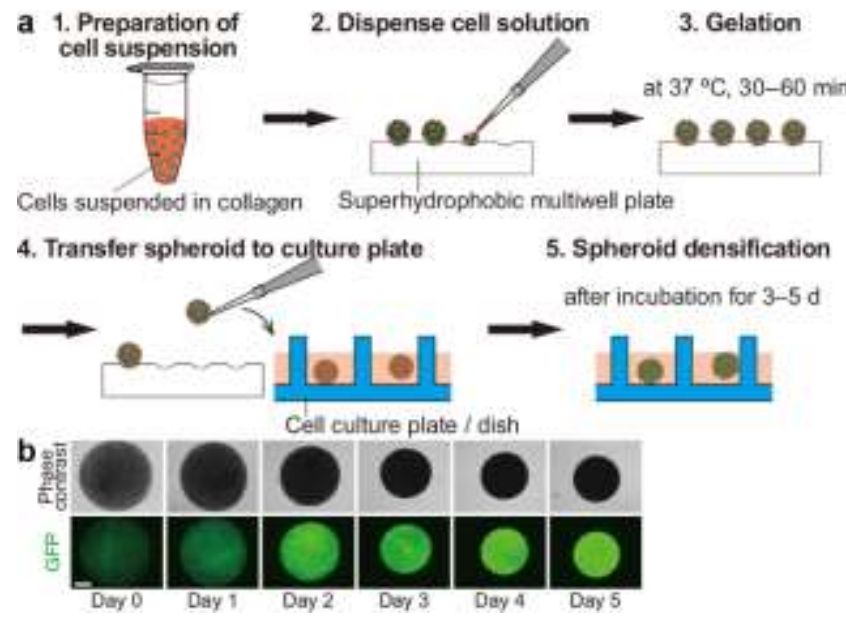

Figure 2. Spheroid formation using the superhydrophobic multiwell plate. (a) A five-step protocol for cell spheroid. (b) Timelapse images of an MDA-MB-231 cancer cell line spheroid formed according to the protocol. The scale bar indicates 500 $\mu \mathrm{m}$.

Using the outlined protocol, we produced spheroids with two types of cancer cells: Green fluorescent protein (GFP)labeled MDA-MB-231 cells and HeLa cells. We then prepared their suspension at the two specified concentrations [ $5 \times 10^{6}$ cells $\mathrm{mL}^{-1}$ (high density) or $5 \times 10^{5}$ cells $\mathrm{mL}^{-1}$ (low density)]. Dulbecco's modified Eagle's medium was used as a cell culture medium. The cell suspension was mixed with $4.2 \mu \mathrm{L}$ of the collagen solution and dispensed onto the multiwell plate to produce spheroids with a diameter of $2 \mathrm{~mm}$. During the 5-day incubation, the cells in the collagen gel sphere grew and increased in density, as confirmed by the increase in the GFP fluorescent intensity (Figure 2b). The increase in cell density in the collagen gel sphere was observed regardless of the cell type or concentration (Figure $3 a, b)$. We then measured the diameter of the formed spheroids based on the captured images with ImageJ $2 .{ }^{24}$ Although their diameter was about $30 \%$ larger than the target diameter of $2 \mathrm{~mm}$, the equivalent spheroids could be produced with an error of less than $10 \%$ (Figure S8a,b, Supporting Information). The spheroid of MDA-MB-231 contracted as the cell density increased, and its contraction speed depended on the concentration of cell suspension. These phenomena were not observed with the HeLa cell 
spheroids or the control collagen gel spheres (Figure 3c, S8c, Supporting Information). The contraction responses were thus unique to MDA-MB-231 cells in the present study, and they have been observed not only in spheroids but also in 3D culture using collagen gel or Matrigel. ${ }^{25,}{ }^{26}$ In addition, the tumor cells started to escape from the spheroid as the cell density increased (Figure $3 \mathrm{~d}$ ). The escaped cells adhered to the bottom surface of the cell culture plate, where they survived and proliferated (Figure 3e).

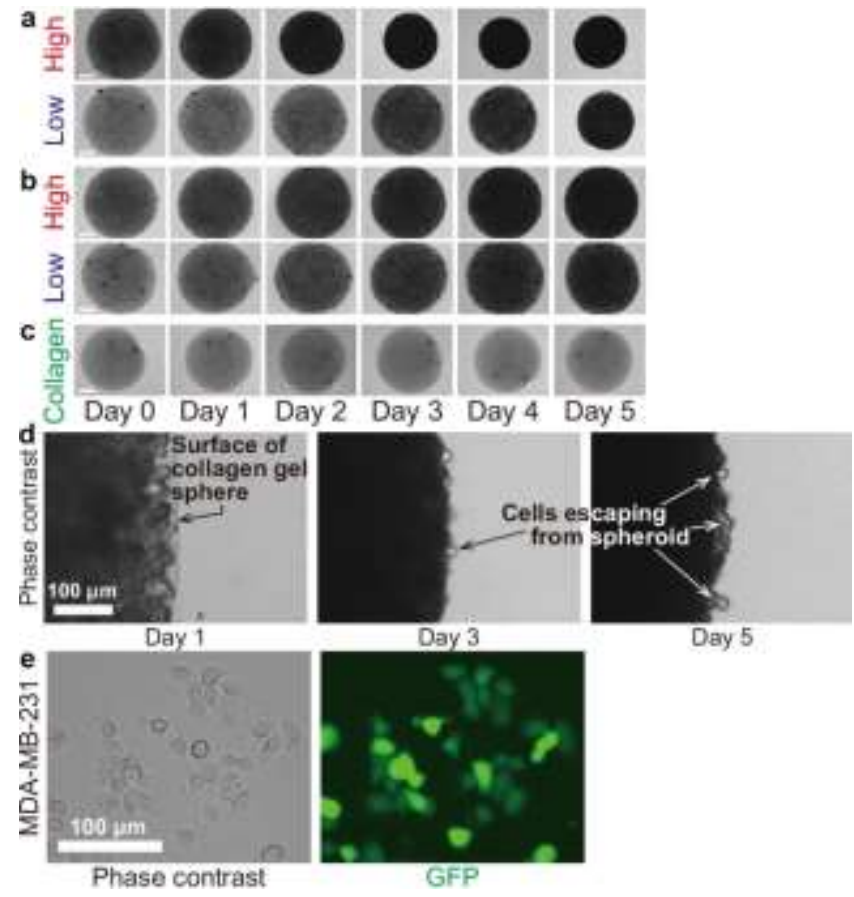

Figure 3. Changes in spheroid dynamics due to the difference in cell suspension concentrations. Phase contrast time-lapse of the spheroids formed with (a) MDA-MB-231 cells, (b) HeLa cells, and (c) collagen gel only. The scale bars indicate $500 \mu \mathrm{m}$. (d) A representative situation of the tumor cells escaping from their spheroid (MDA-MB-231 cells). (e) Representative images of MDA-MB-231 cells, which escaped from the spheroid and adhered to the bottom surface of the cell culture plate after 5 -day incubation.

In summary, we fabricated a low-density macroporous monolith with sufficient strength for CNC milling using a simple sol-gel process. The machined surface of the BNFPMSQ material was found to be superhydrophobic, resulting in droplet retention on appropriately designed shapes. With the CNC milled multiwell plate, we formed 3D cell cultures to obtain high yields of spheroids using two cell types. This system has the potential to be applied to scaffolds to construct 3D tissues (i.e., organoid). We are currently aiming to establish a 3D processing technology with an accuracy of $10 \mu \mathrm{m}$, which will be applied to cell engineering.

\section{ASSOCIATED CONTENT}

\section{Supporting Information}

The Supporting Information is available free of charge on the ACS Publications website.

Experimental procedures, properties of BNF-PMSQ monoliths, microstructure of BNF-PMSQ monoliths, mechanical properties of BNF-PMSQ monoliths, SEM images of CNC-milled flat surface, superhydrophobicity, schematics of the microneedle array and multiwell plate, changes in diameter of spheroids (PDF) Microstructure of a BNF-PMSQ macroporous monolith (MP4)

Time-lapse movie during CNC milling (MP4)

Forming Janus-water droplets on a water-repellent microneedle array fabricated by CNC milling (MP4)

\section{AUTHOR INFORMATION}

\section{Corresponding Author}

* Email: gen@aerogel.jp (G. H.)

* Email: dyoshino@go.tuat.ac.jp (D. Y.)

Notes

The authors declare no competing financial interest.

\section{ACKNOWLEDGMENT}

We are grateful to Dr. Yutaro Hirai (Tohoku University) for his help in taking TEM tilt images. Thank you to the Cell Resource Center for Biomedical Research, Institute of Development, Aging and Cancer, Tohoku University, Japan for providing the cells used in this study. This research was supported by JSPS KAKENHI No. 17K14541, MEXT LEADER Grant, and Takeda Science Foundation.

\section{REFERENCES}

(1) Feinle, A.; Elsaesser, M. S.; Husing, N., Sol-gel Synthesis of Monolithic Materials with Hierarchical Porosity. Chem. Soc. Rev. 2016, 45, 3377-3399.

(2) Plieva, F. M.; Galaev, I. Y.; Mattiasson, B., Macroporous Gels Prepared at Subzero Temperatures as Novel Materials for Chromatography of Particulate-Containing Fluids and Cell Culture Applications. J. Sep. Sci. 2007, 30, 1657-1671.

(3) Monolithic Silicas in Separation Science: Concepts, Syntheses, Characterization, Modeling and Applications; Unger, K. K., Tanaka, N., Machtejevas, E., Eds.; Wiley-VCH Verlag GmbH: Weinheim, 2011.

(4) Liu, T. Q.; Chien, C. C.; Parkinson, L.; Thierry, B., Advanced Micromachining of Concave Microwells for Long Term OnChip Culture of Multicellular Tumor Spheroids. ACS Appl. Mater. Interfaces 2014, 6, 8090-8097.

(5) Guckenberger, D. J.; de Groot, T. E.; Wan, A. M. D.; Beebe, D. J.; Young, E. W. K., Micromilling: A Method for Ultra-Rapid Prototyping of Plastic Microfluidic Devices. Lab. Chip. 2015, 15, 23642378.

(6) Nagai, N.; Mizukami, F., Properties of Boehmite and $\mathrm{Al}_{2} \mathrm{O}_{3}$ Thin Films Prepared from Boehmite Nanofibres. J. Mater. Chem. 2011, 21, 14884-14889.

(7) Hayase, G.; Nonomura, K.; Kanamori, K.; Maeno, A.; Kaji, H.; Nakanishi, K., Boehmite Nanofiber-Polymethylsilsesquioxane Core-Shell Porous Monoliths for a Thermal Insulator under Low Vacuum Conditions. Chem. Mater. 2016, 28, 3237-3240.

(8) Hayase, G., Pseudoboehmite Nanorod-Polymethylsilsesquioxane Monoliths Formed by Colloidal Gelation. J. Asian Ceram. Soc. 2019, 7, 469-475.

(9) Hayase, G.; Kugimiya, K.; Ogawa, M.; Kodera, Y.; Kanamori, K.; Nakanishi, K., The Thermal Conductivity of Polymethylsilsesquioxane Aerogels and Xerogels with Varied Pore Sizes for Practical Application as Thermal Superinsulators. J. Mater. Chem. A 2014, 2, 6525-6531.

(10) Hayase, G., Fabrication of Boehmite Nanofiber Internally-Reinforced Resorcinol-Formaldehyde Macroporous Monoliths for Heat/Flame Protection. ACS Appl. Nano Mater. 2018, 1, 5989-5993.

(11) Xiang, H. F.; Zhang, L.; Wang, Z.; Yu, X. L.; Long, Y. H.; Zhang, $\mathrm{X}$. L.; Zhao, N.; Xu, J., Multifunctional 
Polymethylsilsesquioxane (PMSQ) Surfaces Prepared by Electrospinning at the Sol-Gel Transition: Superhydrophobicity, Excellent Solvent Resistance, Thermal Stability and Enhanced Sound Absorption Property. J. Colloid Interface Sci. 2011, 359, 296-303.

(12) Asghar, W.; El Assal, R.; Shafiee, H.; Pitteri, S.; Paulmurugan, R.; Demirci, U., Engineering Cancer Microenvironments for in vitro 3-D Tumor Models. Mater. Today 2015, 18, 539553.

(13) Lin, R. Z.; Chang, H. Y., Recent Advances in Three - Dimensional Multicellular Spheroid Culture for Biomedical Research. Biotechnol. J. 2008, 3, 1172-1184.

(14) Lawrenson, K.; Sproul, D.; Grun, B.; Notaridou, M.; Benjamin, E.; Jacobs, I. J.; Dafou, D.; Sims, A. H.; Gayther, S. A., Modelling Genetic and Dlinical Heterogeneity in Epithelial Ovarian Cancers. Carcinogenesis 2011, 32, 1540-1549.

(15) Luca, A. C.; Mersch, S.; Deenen, R.; Schmidt, S.; Messner I.; Schafer, K. L.; Baldus, S. E.; Huckenbeck, W.; Piekorz, R. P.; Knoefel, W. T.; Krieg, A.; Stoecklein, N. H., Impact of the 3D Microenvironment on Phenotype, Gene Expression, and EGFR Inhibition of Colorectal Cancer Cell Lines. Plos One 2013, 8, e59689.

(16) Nath, S.; Devi, G. R., Three-Dimensional Culture Systems in Cancer Research: Focus on Tumor Spheroid Model. Pharmacol. Ther. 2016, 163, 94-108.

(17) Kelm, J. M.; Timmins, N. E.; Brown, C. J.; Fussenegger, M.; Nielsen, L. K., Method for Generation of Homogeneous Multicellular Tumor Spheroids Applicable to a Wide Variety of Cell Types. Biotechnol. Bioeng. 2003, 83, 173-180.

(18) Kuo, C. T.; Wang, J. Y.; Lin, Y. F.; Wo, A. M.; Chen, B. P. C.; Lee, H., Three-Dimensional Spheroid Culture Targeting Versatile Tissue Bioassays Using a PDMS-Based Hanging Drop Array. Sci. Rep. 2017, 7, 4363.

(19) Haisler, W. L.; Timm, D. M.; Gage, J. A.; Tseng, H.; Killian, T. C.; Souza, G. R., Three-Dimensional Cell Culturing by Magnetic Levitation. Nat. Protoc. 2013, 8, 1940-1949.

(20) Moghadas, H.; Saidi, M. S.; Kashaninejad, N.; Kiyoumarsioskouei, A.; Nguyen, N. T., Fabrication and characterization of low-cost, bead-free, durable and hydrophobic electrospun membrane for 3D cell culture. Biomed. Microdevices 2017, 19, 74.

(21) Marimuthu, M.; Rousset, N.; St-Georges-Robillard, A.; Lateef, M. A.; Ferland, M.; Mes-Masson, A. M.; Gervais, T., Multi-size spheroid formation using microfluidic funnels. Lab. Chip. 2018, 18, 304-314.

(22) Fu, J. J.; Zhou, Y.; Shi, X. X.; Kang, Y. J.; Lu, Z. S.; Li, Y.; Li, C. M.; Yu, L., Spontaneous formation of tumor spheroid on a hydrophilic filter paper for cancer stem cell enrichment. Colloids Surf. B 2019, 174, 426-434.

(23) Park, J.; Lim, H.; Kim, H. Y., Shape of a Large Drop on a Rough Hydrophobic Surface. Phys. Fluids 2013, 25 (2).

(24) Rueden, C. T.; Schindelin, J.; Hiner, M. C.; DeZonia, B. E.; Walter, A. E.; Arena, E. T.; Eliceiri, K. W., ImageJ2: ImageJ for the Next Generation of Scientific Image Data. BMC Bioinformatics 2017, $18,529$.

(25) Poincloux, R.; Collin, O.; Lizarraga, F.; Romao, M.; Debray, M.; Piel, M.; Chavrier, P., Contractility of the Cell Rear Drives Invasion of Breast Tumor Cells in 3D Matrigel. Proc. Natl. Acad. Sci. U.S.A. 2011, 108, 1943-1948.

(26) Yoshino, D.; Funamoto, K., Oxygen-Dependent Contraction and Degradation of the Extracellular Matrix Mediated by Interaction Between Tumor and Endothelial Cells. AIP Adv. 2019, 9, 045215 . 
ToC

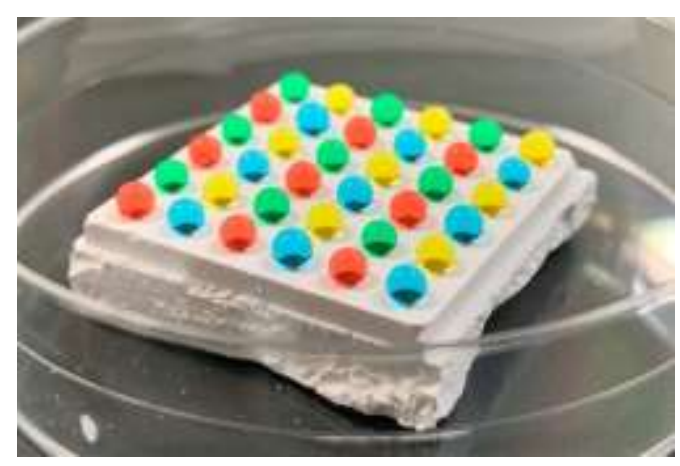

